\title{
Conversor de formatos de arquivos de ferramentas de modelagem de Sistemas a Eventos Discretos
}

\author{
Alberthus Koops Londello \\ athuskoops@yahoo.com.br \\ Universidade Federal de Santa Catarina - Campus Joinville \\ Joinville, SC
}

\begin{abstract}
The project of Discrete Event Systems (DES) with the Supervisory Control Theory uses a group of operations whose algorithms are implemented in tools to support the development of DES. Several tools were developed, each one with self-resources, self-file format and minimal integration between these different tools. This paper presents a tool proposal to convert different files of some tools for the development of DES, using a standard intermediated format defined to the proposal.
\end{abstract}

\section{PALAVRAS-CHAVE}

Conversão de arquivos, Sistemas a Eventos Discretos, Teoria de Controle Supervisório.

\section{INTRODUÇÃO}

Técnicas para o controle de sistemas diferem de acordo com o tipo do sistema a ser tratado. Uma dessas técnicas é a de Sistema a Eventos Discretos (SED), que são sistemas cuja mudança de estado é dependente da ocorrência de eventos em qualquer instante de tempo e seus modelos são obtidos por diversos formalismos [4]. No contexto de aplicações de SEDs, os modelos desenvolvidos são utilizados para projetar controladores, sob forma de supervisores, que irão restringir as dinâmicas do sistema para que eles respeitem diversos requisitos, como segurança e consistência [3]. Para projetar os supervisores, a Teoria de Controle Supervisório (TCS) proposta por [7] apresenta procedimentos formais para a síntese dos supervisores no âmbito dos SEDs.

Para auxiliar no projeto de SEDs, diversas ferramentas foram desenvolvidas para efetuar as computações associadas com os autômatos. Devido ao fato das ferramentas possuírem recursos únicos entre si, o projeto de um sistema complexo de SED pode exigir a utilização de mais de uma dessas ferramentas e, uma vez possuindo diferentes formatos de arquivo para armazenamento dos autômatos gerados nessas ferramentas, é preciso muitas vezes replicar os autômatos do sistema em diversas dessas ferramentas. Em outras palavras, existe pouca ou nenhuma interoperabilidade entre as ferramentas.

Diversas ferramentas foram desenvolvidas para o projeto de SEDs. Este trabalho destaca as ferramentas DESUMA [8], DESTool [1], Nadzoru [6] e IDES [9] para ilustrar diferenças e justificar o trabalho, embora existam outras ferramentas. De maneira geral as ferramentas permitem especificar os autômatos com representação gráfica e realizar as operações básicas de SEDs, como a composição síncrona, e da TCS, como realizar o cálculo do supervisor. Mesmo possuindo recursos similares, ferramentas se destacam por seus recursos diferenciais.

\author{
Benjamin Grando Moreira \\ benjamin.grando@ufsc.br \\ Universidade Federal de Santa Catarina - Campus Joinville \\ Joinville, SC
}

Nesse sentido se propõem uma ferramenta para conversão de arquivos entre diversas ferramentas para SED. Para não realizar uma conversão direta entre todas as diferentes ferramentas, o trabalho propõem um formato intermediário e baseado em XML (Extensible Markup Language).

\section{FUNDAMENTAÇÃO TEÓRICA}

Esta seção apresenta algumas ferramentas para o projeto de SEDs e seus recursos que se destacam. Para desenvolver a conversão de arquivos entre as diversas ferramentas é proposto um formato intermediário baseado em XML.

\subsection{Ferramentas para projetos de SEDs}

A ferramenta IDES (Integrated Discrete-Event Systems) foi desenvolvida na Queen's University e possui uma interface projetada para mimetizar o desenho dos autômatos realizado com "lápis-epapel”. Ela permite a exportação para outros formatos de arquivos de ferramentas SED e também para LateX. A ferramenta suporta a verificação de resultados, como encontrar contra-exemplos e determinar se determinada cadeia de eventos pode ser reconhecida pelos autômatos. Também permite a geração automática de código [9].

DESUMA é uma ferramenta resultante da integração da biblioteca UMDES (biblioteca para estudo de SEDs desenvolvida pela Universidade de Michigan), com a interface gráfica GIDDES (Graphical Interface for the Design of Discrete Event Systems - desenvolvida pela Universidade de Mount Allison). A ferramenta DESUMA implementa diversas técnicas para a construção de diagnosticadores de falha para SEDs, incluindo rotinas para construir e analisar autômatos diagnosticadores [8].

A ferramenta Nadzoru permite realizar a simulação dos autômatos, acompanhando de forma animada a transição entre os estados quando determinado evento ocorre. A Nadzoru também permite a geração de código para múltiplos dispositivos (geração de código para dispositivos diferentes daqueles disponíveis na ferramenta IDES) e também permite a análise de problemas relacionados com a lógica de controle associada com a implementação do autômato supervisor [6].

Por fim, a ferramenta DEStool é uma interface gráfica para a biblioteca libFAUDES, sendo essa uma biblioteca escrita em $\mathrm{C}++$ e de código aberto. Além de diversas operações relacionadas com SEDs e TCS que não existentes nas outras ferramentas, a DESTool suporta a especificação de scripts em linguagem de programação Lua, o que facilita a realização de diversas rotinas [1]. 


\subsection{XML}

Extensible Markup Language (XML) é um formato de texto simples é flexível. A XML foi projetada para publicação eletrônica e para lidar com desafios de troca de dados [2].

Um arquivo XML possui em seus dados as informações pertinentes ao arquivo que se quer representar especificamente. Para um modelo que define a estrutura/formato de especificação do arquivo XML é estabelecido um XML Schema.

\section{SOLUÇÃO PROPOSTA}

A proposta desse trabalho consiste em especificar um XML Schema para ser utilizado como formato intermediário na conversão entre diversos formatos de arquivos gerados por ferramentas para projetos de SEDs.

Para realizar a conversão direta entre as 4 ferramentas citadas no trabalho, é preciso elaborar 3 scripts de conversão de um formato para os outros 3 formatos, ou seja, 12 scripts de conversão para converter os arquivos entre as 4 ferramentas. Com a utilização do XML intermediário proposto, são necessários 4 scripts para converter cada formato para o XML e outros 4 scripts para converter do XML para o formato de arquivo desejado, ou seja, 8 scripts. As Figuras 1 e 2, ilustram essa questão.

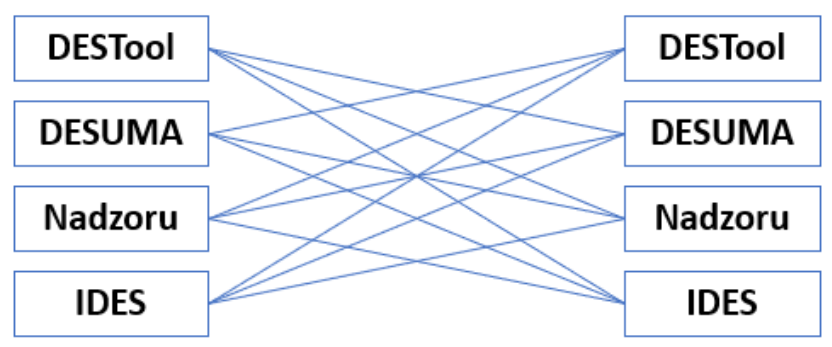

Figura 1: Conversão direta entre os formatos de arquivo

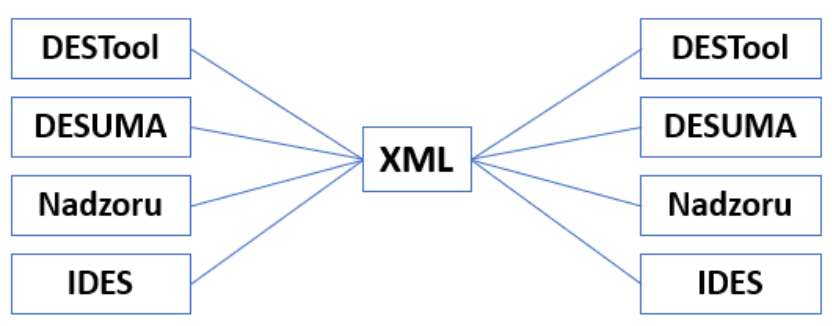

Figura 2: Conversão entre os formatos de arquivo utilizando um formato intermediário

Se o suporte de conversão for para o dobro de ferramentas, então seriam necessários 56 scripts de conversão sem o formato intermediário e 16 scripts utilizando o formato intermediário, uma nítida vantagem da abordagem utilizando o formato intermediário.

Embora as ferramentas para o projeto SEDs possuam recursos próprios e distintos, o arquivo gerado por elas deve armazenar os autômatos projetados e calculados através das operações. Nesse sentido, foi identificado que o formato do arquivo das diversas ferramentas precisam especificar alguns elementos em comum, como:

- Estados do autômato: determinar quais estados e se os estados são estados marcados ou não.

- Alfabeto de eventos: determinar se o evento é controlável e/ou observável.

- Transições existentes: indicar origem, destino e evento da transição.

Sendo assim o trabalho objetiva definir o XML Schema para representar os autômatos e elaborado scripts de conversão de diversas ferramentas para o XML definido e vice-versa.

\section{CONSIDERAÇÕES FINAIS}

O trabalho teve início com o mapeamento dos elementos e recursos utilizados por cada ferramenta para definir os autômatos. Algumas propriedades do autômato são únicas de algumas ferramentas. Por exemplo, a ferramenta DESTool especifica uma propriedade denominada "forçável" para os eventos. O arquivo XML deverá considerar essa propriedade, mesmo que ela não seja utilizada no formato de arquivo de destino.

Uma informação armazenada nos arquivos e que será desconsiderada inicialmente no trabalho é o posicionamento dos estados e transições. Essa é uma questão estética, embora importante. As ferramentas utilizam a biblioteca GraphViz [5] para a representação gráfica do autômatos, sendo que esta biblioteca possui algoritmos para organização automática e posicionamento de estados e transições.

Além das inúmeras ferramentas para SEDs, também existem outros formatos interessantes para especificação, como LateX e da própria GraphViz.

\section{REFERÊNCIAS}

[1] [n.d.]. DESTool: about. https://fgdes.tf.fau.de/destool/. Acessado em: 2019-10-21.

[2] [n.d.]. Extensible Markup Language (XML). https://www.w3.org/XML/. Acessado em: 2019-10-21.

[3] John Baillieul and Tariq Samad. 2015. Encyclopedia of systems and control. Springer Publishing Company, Incorporated.

[4] Christos G Cassandras and Stephane Lafortune. 2009. Introduction to discrete event systems. Springer Science \& Business Media.

[5] John Ellson, Emden Gansner, Lefteris Koutsofios, Stephen C North, and Gordon Woodhull. 2001. Graphviz-open source graph drawing tools. In International Symposium on Graph Drawing. Springer, 483-484.

[6] Lucas Preischadt Pinheiro, Yuri Kaszubowski Lopes, André Bittencourt Leal, and Roberto Silvio Ubertino Rosso Junior. 2015. Nadzoru: A software tool for supervisory control of discrete event systems. IFAC-PapersOnLine 48, 7 (2015), 182-187.

[7] Peter JG Ramadge and W Murray Wonham. 1989. The control of discrete event systems. Proc. IEEE 77, 1 (1989), 81-98.

[8] Laurie Ricker, Stephane Lafortune, and S Genc. 2006. Desuma: A tool integrating giddes and umdes. In 2006 8th International Workshop on Discrete Event Systems. IEEE, 392-393.

[9] Karen Rudie. 2006. The integrated discrete-event systems tool. In 2006 8th International Workshop on Discrete Event Systems. IEEE, 394-395. 